beschreibt der Autor drei Arten der Produktivität von Fans: die semiotische, die expressive und die textuelle. Diese Differenzierung ist in der Auseinandersetzung mit der Produktivität der Zuschauer, vor allem in der Kritik daran, häufig verloren gegangen. Zusammenfassend stellt der Autor fest, ",dass die Fans Filme anders interpretieren und gebrauchen, als dies Außenstehende tun (können). Das jeweils verfügbare Wissen, interpretative Strategien, das gemeinschaftliche Erleben oder die Abgrenzung gegen Nicht-Fans führen zu unterschiedlichen $R a b-$ mungen und Gebrauchsweisen der Filme“ (S. 287). Nicht nur Fans, sondern alle Zuschauer versuchen in der Rezeption von Filmen aus etwas Vorgegebenem etwas Eigenes zu machen. Darin sieht Winter das Wesen von Medienaneignung als kulturellem und ästhetischem Prozess.

Der Neuauflage ist ein Nachwort beigefügt, in dem sich der Autor mit „Perspektiven und Problemen der aktuellen Fanforschung “ (S. 288ff.) auseinandersetzt. Dabei unterscheidet er drei Phasen der Fanforschung im Kontext von akademischer Kultur und Fankultur. Die digitale Transformation von Fankulturen wird in einem weiteren Abschnitt behandelt. Darin stellt Winter z. B. im Anschluss an Henry Jenkins fest, dass Quality TV „ohne die Aktivitäten der Fans im Internet nicht denkbar" sei (S. 303). Allerdings klassifiziert er diese Feststellung als optimistisch, weil sie „die konkreten Macht- und Herrschaftsverhältnisse in der globalen Postmoderne" ausblende (ebd.). Der zwar differenzierten, aber doch sehr optimistischen Sichtweise des Autors in der Erstauflage, in der die Kreativität und Produktivität der Fans deutlich betont wurden, ist in der Neuauflage einer etwas skeptischeren Sichtweise gewichen: „Dabei verkörpern Fankulturen wie auch die Populärkultur im 21. Jahrhundert nicht zwangsläufig progressive Werte. Sie stehen in enger Interaktion mit den transnationalen Kulturindustrien, die die Machtverhältnisse in der globalen Postmoderne nicht grundsätzlich infrage stellen. Nichtsdestotrotz stellt die Populärkultur einen sich entwickelnden und differenzierenden Bereich der Interaktion und des Aushandelns von Sinn dar, dessen Bedeutung notwendigerweise ambivalent bleibt" (S. 305). Und, könnte man hinzufügen: Diese Ambivalenz macht gerade die Stärke der Populärkultur aus, denn so lässt sie sich nicht gänzlich vereinnahmen. Es bleibt immer ein (Rest von) Eigensinn.

Die neuerliche Lektüre des Bandes macht deutlich, dass es sich - auch wenn es im Kontext der Cultural Studies zu verorten ist - um einen
Klassiker der Medien- und Kommunikationswissenschaft handelt. Mit dem Buch hat Rainer Winter Mitte der 1990er Jahre des 20. Jahrhunderts die theoretischen und empirischen Grundlagen der Fanforschung im deutschsprachigen Raum gelegt. Der Autor reflektiert selbst, dass einige der damaligen Erkenntnisse im historischen Kontext gesehen werden müssen. Das mindert jedoch nicht die weitreichende Bedeutung, die dieses Buch hatte und mit der Neuauflage immer noch hat. Gerade die dichte Beschreibung der Fankultur der Horrorfans macht deutlich, wie wichtig differenzierte Sichtweisen auf die kulturellen und ästhetischen Praktiken (nicht nur) von Fans sind.

Lothar Mikos

\section{Rainer Winter}

\section{Widerstand im Netz}

Zur Herausbildung einer transnationalen

Öffentlichkeit durch netzbasierte

Kommunikation

Bielefeld: Transcript, 2010. - 165 S.

(Cultural Studies; 21)

ISBN 978-3-89942-555-0

Inwieweit mit der zunehmenden Verbreitung des Internets neue Formen transnationaler bzw. transkultureller Öffentlichkeit möglich sind bzw. ob diese zu einer Erstarkung der Zivilgesellschaft beitragen, ist ein Thema, das seit Längerem die Kommunikations- und Medienwissenschaft beschäftigt. In einen solchen Diskurs fügt sich auch das rund 150-seitige Bändchen von Rainer Winter ein. Dessen Grundlage ist ein gemeinsam mit Sonja Kutschera-Groinig für das Büro für Technikfolgenabschätzung des Deutschen Bundestags im Jahr 2004 verfasstes Gutachten. Wie der Titel schon suggeriert, geht es in dem Band darum, das in solchen Zusammenhängen bestehende Potenzial von „Widerstand" aus Sicht der Cultural Studies auszuloten.

Das Buch selbst gliedert sich in sieben Kapitel. Nach einer kurzen Einleitung wird in Kapitel 2 der Zugang der Cultural Studies zu digitalen Medien und der Herausbildung einer netzbasierten Öffentlichkeit dargelegt. Es geht hier insbesondere um die Potenziale von ,agency" in der Netzkommunikation bzw. die Frage, inwieweit „widerständige digitale Praktiken“ bestehen (können). Kapitel 3 befasst sich dann mit der kommunikativen und kulturellen $\mathrm{Di}^{-}$ mension des Internets. Nach einer kurzen Hinführung zur Geschichte des Internets wird insbesondere diskutiert, inwieweit sich im bzw. 
durch das Internet Prozesse kultureller Hybridisierung gefördert werden, die es in einer (an Ulrich Beck angelehnten) kosmopolitischen Perspektive zu analysieren gilt. Auf dieser Basis werden Markt, Staat und Zivilgesellschaft als die drei zentralen Akteure der Netz-Öffentlichkeit behandelt. Das Kapitel 4 stellt letztlich einen Exkurs dar, der sich weniger mit der transnationalen Öffentlichkeit im Internet befasst denn mit der Transformation von Fankulturen $\mathrm{zu}$ „electronic tribes“. Kapitel 5 kehrt dann wieder zum Hauptthema des knappen Buchs zurück, nämlich der demokratischen Öffentlichkeit im Internet. Hier werden als Erstes ausgehend vom deliberativen Öffentlichkeitsmodell verschiedene Ansätze der Beschreibung vernetzter (Gegen)Öffentlichkeit bzw. der „digital citizenship“ diskutiert. Zugespitzt wird dies in einer Behandlung des „Kampf[s] um eine andere Globalisierung“ (S.101ff.). Kapitel 6 stellt dann unter der Überschrift „transnationaler Aktivismus und zivilgesellschaftliche Bewegungen“ eine Art Analyse von drei „zivilgesellschaftlichen virtuellen Netzwerken" dar (Association for Progressive Communications, Friends of the Earth International und OneWorld) sowie des Online-Portals der EU. Die Schlussfolgerungen des Kapitels 7 fassen die verschiedenen Analysen nochmals in dem Sinne zusammen, dass „das Internet die Artikulation unterschiedlicher alternativer Stimmen, Positionen und Perspektiven erlaubt" (S. 145), weswegen es „wichtig [ist], kosmopolitische Perspektiven zu stärken und zu forcieren sowie eine globale Demokratie als Zielsetzung zu haben“" (S. 146).

Versucht man dieses wie gesagt vergleichsweise knappe Buch insgesamt zu würdigen, so besteht seine Leistung vor allem in seinen referierenden Passagen. In diesen werden verschiedene jüngere Arbeiten von Vertreterinnen und Vertretern der Cultural Studies (und diesen nahe stehenden Traditionen) aufgearbeitet, die „(digitale) Medienkulturen innerhalb sozialer Bewegungen und alternativer Gemeinschaften untersuch[en], und auch erforsch[en], wie sie durch die Kommunikationen in Gemeinschaften und Bewegungen erst erschaffen werden" (S. 38). Solche Darstellungen machen deutlich, welches Potenzial gerade die Cultural Studies für eine Forschung zu alternativer Öffentlichkeit und kommunikativer Vernetzung im Internet haben.

Leider fällt das Buch deutlich ab, wo es um eigene Analysen geht. Insbesondere das Kapitel 7 ist nicht mehr als eine systematisierte Darstellung der Webseiteninhalte der betrachteten (zivilgesellschaftlichen) Organisationen. Eine weitergehende und vertiefende Forschung, wie sie eigentlich aus den empirischen Analysen der Cultural Studies bekannt ist, unterbleibt ebenso wie eine methodische Reflexion des Vorgehens. An dieser Stelle ist das Buch dann doch zu sehr Gutachten und zu wenig empirische Forschung. Dieser Gutachtencharakter tritt daneben auch an anderen Stellen in den Vordergrund, wenn bspw. in Bezug auf den Charakter des Internets eher salopp und auch widersprüchlich argumentiert wird. So heißt es etwa zum Internet bzw. WWW anfangs, dass „keine stabilen sozialen und kulturellen Bedeutungen sowie Gebrauchsweisen [...] aus ihren inhärenten (materiellen) Eigenschaften einfach ableitbar wären" (S. 16). Später finden sich dann allerdings Formulierungen wie, dass sich das Internet bzw. WWW „als eine kulturelle Technologie begreifen [lässt], die eine eigene räumliche Logik der Produktion, Verbreitung und des Konsums hervorgebracht hat" (S. 19), eine „vernetzte, dezentrale Logik“ (S. 29). Hier hätte man sich deutlich mehr begriffliche Schärfe gewünscht, die bei Vertreterinnen und Vertretern der Cultural Studies durchaus zu finden ist, wo doch bspw. Autoren wie David Morley oder Nick Couldy für eine sehr differenzierte Annäherung an digitale Medien argumentieren, die diesen gerade nicht kontextfrei bestimmte Logiken unterstellt, oder Kevin Robins sehr früh zu einer vorsichtigen und kritischen Analyse des „Cyberspace“ aufgerufen hat.

Insofern empfiehlt sich der Band weniger als empirische „cultural study“ im ursprünglichen Sinne des Wortes, sondern vielmehr als ein knappes Buch, das einen Einstieg in den Blickwinkel der Cultural Studies auf transnationale Öffentlichkeit im Internet erleichtert. Erwartet man dies, so lohnt die bisweilen kurzweilige Lektüre.

Andreas Hepp 